\title{
Protée
}

\section{Le bon ange de la certitude. À l'origine du sujet et du nom chez Proust}

\section{Jacques Cardinal}

Volume 28, numéro 1, 2000

Variations sur l’origine

URI : https://id.erudit.org/iderudit/030585ar

DOI : https://doi.org/10.7202/030585ar

Aller au sommaire du numéro

\section{Éditeur(s)}

Département des arts et lettres - Université du Québec à Chicoutimi

ISSN

0300-3523 (imprimé)

1708-2307 (numérique)

Découvrir la revue

Citer cet article

Cardinal, J. (2000). Le bon ange de la certitude. À l'origine du sujet et du nom chez Proust. Protée, 28(1), 75-93. https://doi.org/10.7202/030585ar
Résumé de l'article

La question de l'origine de la conscience se pose chez Proust depuis une expérience singulière de la mémoire. En analysant la poétique, l'imaginaire et l'enjeu symbolique de la nomination, l'auteur montre que l'origine n'est pas que vertige et oubli, mais expérience de la consistance et de quelque ponctuelle certitude. L'analyse permet d'expliciter aussi la logique du signifiant, c'est-à-dire ce qui, depuis l'origine de ces premières traces de désir, ne cesse de hanter la parole du sujet. 


\section{LE BO N AN GE DE LA CERTITU DE À L'ORIGINE DU SUJET ET DU NOM CHEZ PROUST}

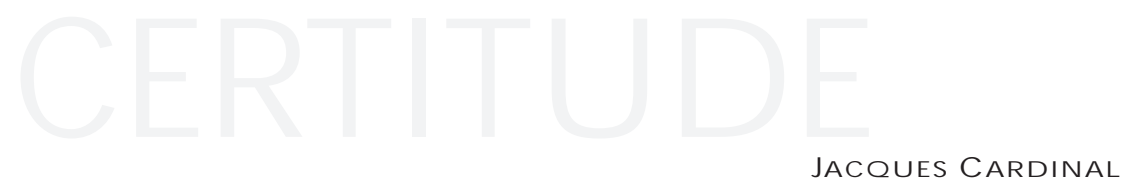

Certains hommes seuls se penchent sur l'inexplicable sillon tracé en eux. Les sillons affectent différentes formes mais toutes obscures comme les signes d'une écriture antique qu'on ne connaîtrait pas. Là un poignard, là un simple signe légèrement brillant: là un rectangle, etc., une fleur. [...] Et pourtant il n'y a d'intelligence vraie, de réalité rationnelle que celle qu'on trouve si on arrive à comprendre ce que signifiait pour nous au moment où le petit sillon fut tracé, le poignard, le clocher, le rectangle ou la fleur. [...] La seule réalité est celle qu'on arrive à dégager des formes inexplicables laissées par ce que nous avons effectivement senti. ${ }^{1}$

Il est assez connu que la question de l'origine chez Proust se pose depuis une conception particulière de la mémoire et de la sensibilité (dont l'épisode de la madeleine, notamment, donne la clé). À cet égard, la Recherche se laisse lire comme une nouvelle phénoménologie de la perception où se dévoile une expérience tout à la fois fragmentée, perspectiviste et pourtant ponctuellement unifiante, de la conscience. Vision impressionniste où se nouent l'objet et le sujet, le temps et l'espace, traçant ainsi le «sillon» d'une émotion ou d'un regard encore indéchiffrable, mais qu'une certaine pratique de l'art pourra à nouveau faire revivre ou recréer. Le sujet proustien se tient ainsi à ce carrefour où s'entrecroisent ce noyau de sensations confuses mais décisives qui le hantent et l'écriture remémorante de ce sentiment qui est comme la vérité de son désir enfin nommé. À l'origine serait ainsi une trace innommée - «obscures comme les signes d'une écriture antique» - dont le corps est le premier livre, et que le sujet ne sait pas lire (encore); l'écriture proustienne s'édifiant sur ce sillon ancien, premier corps d'écriture dont l'énigme détermine le sujet. Ce n'est donc pas la primauté de la conscience qui est ici supposée, mais l'antériorité originaire du sentiment et du corps, depuis lesquels cependant s'élabore l'avènement du sujet (et ce qu'il assumera enfin de ce sentiment, de ce «sillon»). Cette antériorité du corps sur la conscience se confond avec ce temps originaire où le parlêtre conserve le vague souvenir - déposée en quelque(s) signifiant(s) maître(s) - d'une communion prétendument béate avec le monde et qui se soutient notamment de l'illusion d'une voix isomorphe aux êtres et aux choses, miroir pur de sa demande 
supposément entendue. Premier corps du sujet qui est égal à son inscription dans la consistance et la jouissance première de ses signifiants; préambule, dans ce cas, ou condition de possibilité de son inscription dans l'ordre symbolique où s'ordonne l'expérience de l'altérité et de l'arbitraire (dont la métaphore paternelle est le support logique). À cet égard, le romanesque proustien se débat aussi avec ce premier temps du sujet, lequel insiste longtemps après comme étant celui où règne le signifiant, en tant qu'il est justement le support d'une jouissance inaugurale, ressurgissant comme cette trace originaire que la reconnaissance de l'arbitraire entre les mots et les choses ne pourra jamais effacer complètement; rêverie mimologique qui suppose en effet le souvenir d'un premier temps où le sujet advient dans sa différence (donc, en son identité) par la profération de quelques signifiants qui le parlent et le nomment, lui donnant ainsi l'être. Cette profération des signifiants instaure d'ailleurs la première scansion entre le plein et le manque, scansion depuis laquelle le sujet tente de maîtriser et de jouir du monde en le parlant. C'est l'inscription de cette première jouissance que le sujet voudra revivre en se faisant plus tard maitre des noms, afin de retrouver en eux quelque chose de la consistance originaire de sa propre venue à l'être.

C'est ce drame du sujet, du langage et de l'origine, que l'on peut déchiffrer dans «Noms de pays: le Nom", dernier chapitre de Du côté de chez Swann. La lecture minutieuse de ce chapitre - que l'on analysera épisode par épisode afin d'expliciter l'ordre actantiel et l'incidence des oppositions structurantes qui le traversent - montre en effet un rapport ambigu du sujet aux noms, alors que le Narrateur, encore enfant, passe d'une vision monochrome à une autre, multiple et complexe, dépassant ainsi la rêverie originaire sur les signifiants depuis le savoir d'une nouvelle expérience où entre en jeu le désir de l'autre. Bref, il s'agit d'un récit où se met en scène le renoncement envers la rêverie première d'une langue motivée - le signifiant étant ici la trace de cette fusion entre l'être et le corps -, en même temps qu'émerge chez le Narrateur une nouvelle conscience de soi et du monde. Or ce processus repose également sur une écriture ou, si l'on veut, une poétique dont il faut rendre compte afin de montrer comment la description s'avère le miroir du sujet. Outre ce passage de la rêverie motivante à la désillusion, un autre passage se dessine en effet qui va d'une esthétique impressionniste à une autre où prédomine le motif géométrique; ce passage du flou à la discernabilité de la ligne n'est sans doute pas fortuit, mais désigne plutôt le cadre formel d'une quête identitaire où le sujet est incertain dans ses contours comme la fumée et, en même temps, consistant comme le cristal. Double inscription et double écriture où se remarquent les deux temps de la conscience.

À cet égard, la poétique du texte proustien est en elle-même une théorie de l'origine et de la subjectivité, l'expérience du Narrateur s'écrivant ici dans l'étoffe d'un style où sont dévoilées les traces secrètes et dispersées de la mémoire.

\section{MURS-MIROIRS}

Le récit de «Nom de pays: le Nom» s'ouvre sur la description de la chambre du Grand Hôtel de la Plage à Balbec; celle-ci apparaît moins comme un espace clos, étanche ou neutre, que le lieu où le sujet expérimente toutes sortes de sensations, alors qu'elle est «[...] saupoudrée d'une atmosphère grenue, pollinisée, comestible et dévote [...] dont les murs passés au ripolin contenaient, comme les parois d'une piscine où l'eau bleuit, un air pur, azuré et salin» (376). La chambre est tout autant jardin de fleurs que piscine, lieu multiple qui s'offre autant aux plaisirs de la dégustation que de l'odorat. L'air n'y est donc pas l'élément ou l'équivalent de toutes les transparences, mais la matière qui recueille et condense de multiples sensations florales et marines pour donner à ressentir ce lieu comme une ouverture sur d'autres lieux. Décloisonnement qui suppose le règne du mélange où l'air, justement, devient "atmosphère» des lieux (et, éventuellement, matière signifiante du temps mémoriel). La suite du texte insiste d'ailleurs sur ce décloisonnement:

Le tapissier bavarois [...] avait varié la décoration des pièces et sur trois côtés fait courir le long des murs [...] des bibliothèques basses, à vitrines en glace, dans lesquelles, selon la place qu'elles 
occupaient [...] telle ou telle partie du tableau changeant de la mer se reflétait, déroulant une frise de claires marines,

qu'interrompaient seuls les pleins de l'acajou. (376)

Les murs-bibliothèques de la chambre semblent ainsi se liquéfier pour mieux absorber le paysage et rompre avec le cloisonnement ordinaire ou supposé tel de l'espace; chambre-piscine, chambre-aquarium, chambre-paysage, murs-miroirs, autant d'enchâssements et de superpositions où le sujet se déplace, transporté par le flux multiple des sensations et des images. La séparation entre le dedans et le dehors s'efface là où le mur est devenu la surface réfléchissante du monde; la dureté du mur se laissant creuser mollement pour donner à voir une autre vision du monde. Le mur est donc le contraire d'un espace neutre, blanc, lisse, opaque ou aveugle; ce mur-miroir étant plutôt comme la page d'un livre - une toile ou un écran de cinéma - sur lequel vient s'écrire le récit fragmentaire d'un monde qui ne cesse de changer à mesure qu'il défile, emporté par le temps. Murbibliothèque qui est aussi une surface d'écriture dont l'éventuelle lecture révélera les paysages et les intrigues; l'aveugle opacité de la paroi s'ouvrant encore sur le voyage imaginaire. On reconnaît dans la description de cette scène l'esthétique impressionniste du texte proustien où, par le jeu de la lumière, les objets s'allument de reflets réciproques (pour le dire comme Mallarmé); effets de condensation des corps qui sont, sur le plan stylistique, rendus par le jeu de la métaphore et de la métonymie ${ }^{2}$.

Toutefois, si le mur - paroi délimitant en principe des espaces distincts - se fait ici miroir, les "pleins de l'acajou " de la bibliothèque sont cependant autant de lignes qui le traversent pour le montrer; effet de coupure et de cadrage venant soutenir un regard qui sans cela pourrait se perdre dans le pluriel des sensations et le fondu des images. Voir, en cela, c'est aussi cadrer, discerner, délimiter. Le sujet ne baigne donc pas dans une totale illusion, mais reste arrimé au monde en vertu d'un certain savoir de l'illusion (puisque ce cadrage permet de reconnaître le mur comme miroir). Les "pleins de l'acajou» sont donc ce par quoi le sujet s'inscrit encore dans l'ordre du discernable, là où l'expérience de l'indiscernabilité - la confusion des lieux et des temps - peut ultimement avoir du sens. Si, donc, l'expérience du monde est d'abord celle d'une certaine déformation illusion, fragmentation, perspectivisme, subjectivisme -, il n'en reste pas moins qu'elle ne se saisit que depuis son rapport au jugement, à l'analyse et à la lucidité (ou à une certaine vérité, celle qui s'inscrit dans le désir et sa loi). La chambre est ainsi, à la fois, fermée et ouverte. De plus, si l'on considère que cet imaginaire est aussi le miroir par lequel se saisit le sujet, on constate que, tout comme dans le cas du mur, il est traversé par ces deux temps où il est tout à la fois poreux et assez consistant en son être pour que cette expérience de relative dissémination puisse être perçue comme telle. Le regard que pose le Narrateur sur la chambre de l'hôtel à Balbec révèle ainsi un tant soit peu la structure du sujet qui est à la fois ouvert au monde, en raison de la plasticité de son imagination, et, cependant, relativement déterminé en raison des circonstances toujours contingentes où son noyau identitaire a dû s'élaborer. Ce noyau identitaire lui est notamment donné par le nom qui l'ancre minimalement, mais non moins fondamentalement, dans le monde. Le nom, en tant que pur signifiant, fait trou (et bord) en effet dans l'indifférencié pour donner forme à la scansion première du même et de l'autre, de l'identité et de la différence, étant, à la manière du trait unaire, ce qui fait qu'il y a de l'UN $\mathrm{N}^{3}$.

Ce foisonnement des images n'est donc pas dissémination absolue, mais multiplicité qui se laisse en partie déchiffrer par le tranchant d'un regard capable de discerner quelques lignes à la surface bariolée des choses. Déchiffrer le monde suppose ainsi le foyer d'un regard depuis lequel est tracée la ligne délimitante qui rompt avec le magma des sensations; première trace d'un ordre ou d'une forme qui fonde la représentation et son sujet.

\section{MORTELLE TEMPÊTE}

Si la description de cette chambre d'hôtel nous place ainsi, d'entrée de jeu, dans le registre de 
l'illusion ou de la multiplicité mouvante des êtres et des choses, il n'en demeure pas moins que le Narrateur nous raconte, à cet égard, un processus de désillusion et de conversion à quelque bon usage de l'imagination. Ainsi commence d'ailleurs le paragraphe qui suit: "Mais rien ne ressemblait moins non plus à ce Balbec réel que celui dont j'avais souvent rêvé [...]» (376). Il s'agit alors pour le Narrateur de mesurer les effets de distorsion de sa rêverie d'enfant sur le monde; voilà pourquoi il en appelle aussi au "réel» afin de ne pas se laisser envahir par la folie. Le texte se construit ainsi sur l'opposition du rêve et de l'éveil, de l'illusion et de la lucidité, sans laquelle sans doute le vertige de l'illusion ne serait pas même senti comme tel. L'appel à la «vie réelle de la nature» est donc l'appel à ce qui, chez le Narrateur, serait plus fort que la rêverie:

Je n'avais pas de plus grand désir que de voir une tempête sur la mer, moins comme un beau spectacle que comme un moment dévoilé de la vie réelle de la nature; ou plutôt il n'y avait pour moi de beaux spectacles que ceux que je savais qui n'étaient pas artificiellement combinés pour mon plaisir, mais étaient nécessaires, inchangeables - les beautés du paysage ou du grand art. (377)

Cette scène apparaît d'abord comme une scène de vérité - de vie ou de mort. N'est-elle pas en effet la scène de l'appel à l'avènement de la loi - expérience qui d'emblée assigne le Narrateur à une contingence existentielle dans la mesure où il y reconnaît non seulement la "vie réelle de la nature", mais une force à laquelle il est assujetti? Tempête qui le fait aussi, du même coup, sujet-de-la-mort ou de la finitude alors qu'il vient là se heurter à une autre vision où la mort apparaît comme la scène ultime qui arrête le déchaînement de l'imagination et son ludisme supposé. Moment de vérité qui coïncide avec l'extrême péril, là où l'ultime maître est la mort (pour le dire avec Lacan) ${ }^{4}$. Appel, donc, au dépassement: «Je n'étais curieux, je n'étais avide de connaître que ce que je croyais plus vrai que moi-même $[. .$.$] » (377). Manière de$ dire encore que la souveraineté imaginaire du Narrateur - et son désir de rêver à l'infini ce que pourrait être le monde - s'arrête ici sur cette scène, ce sublime tableau devant lequel il éprouve sa finitude ou sa contingence. La nature tempétueuse et mortelle suppose en effet cette altérité sur laquelle vient se briser la toute-puissance du sujet-de-la-rêverie, altérité qui s'avère «l'absolument vrai»: «Je voulais aussi pour que la tempête fût absolument vraie, que le rivage luimême fût un rivage naturel, non une digue récemment créée par une municipalité» (377). Bien que la scène soit racontée sur un ton où s'entend la naïveté propre à l'enfance, elle n'en reste pas moins structurante dans le jeu de cette opposition entre l'intériorité omnipotente de la rêverie et la contingence de l'extériorité où se révèle l'expérience de l'autre (selon toutes ses formes). Ainsi, de la chambre protéiforme à la tempête comme scène ultime de vérité, un parcours narratif et imaginaire se dessine qui coïncide avec la quête identitaire du Narrateur.

Le réveil du sujet réside ainsi non seulement dans le fait de se savoir mortel, mais de le savoir en renonçant du coup à la toute-puissance de la rêverie qui, ignorant les contingences de l'espace-temps, donne l'illusion de se réfugier dans l'éternité ou l'immortalité. Se savoir mortel - quand bien même la mort serait irreprésentable -, ce n'est en définitive que le savoir inconscient d'être entièrement déterminé par le manque, l'interdit et la contingence, à partir de quoi désirer prend un sens. À l'origine, serait donc aussi ce nœud de désir et de mort qui fonde la vie. D'une scène à l'autre - murs-miroirs et tempête -, le Narrateur passe ainsi des jeux de l'illusion à l'appel véhément de la vérité; non pas pour se neutraliser dans sa quête de savoir, mais pour mieux en reconnaître les vertiges et les certitudes.

\section{LA LIGNE DU TRAIN}

Commence ensuite le long développement sur le nom. On remarque d'abord que la rêverie du Narrateur sur Balbec a pour point de départ un effet de citation; c'est Legrandin et Swann qui ont d'abord nommé et décrit Balbec et qui l'ont ainsi donné à désirer. Le nom surgit donc avec l'aura de sa renommée qui, de surcroît, enchaîne le sujet à 
l'Histoire; car cet enchaînement est déjà le fil de l'Histoire ou l'anastomose du récit où s'inscrit le Narrateur. Manière encore de dire qu'il n'a pas un accès immédiat au monde, mais qu'il est plutôt toujours déjà pris dans une chaîne (une généalogie) dont il ne connaît pas (encore) l'enchaînement singulier. Quelques pages plus loin, le Narrateur est d'ailleurs explicite à cet égard:

Aller aux Champs-Élysées me fut insupportable. Si seulement

Bergotte les eût décrits dans un de ses livres, sans doute j'aurais

désiré de les connaître, comme toutes les choses dont on avait

commencé par mettre le «double» dans mon imagination. (386)

C'est ce «double» qui est premier, et le monde ne se donne au sujet que nommé, toujours déjà. Division inaugurale qui détermine son expérience du temps et de l'espace; division qui le fait sujet à la fois de la perte et de la quête. Au commencement, est donc aussi la re-nommée, c'est-à-dire la répétition, dans la mesure où connaître n'a lieu que depuis le désir de cet autre qui nous parle déjà en nous dévoilant le monde; le premier regard que pose le sujet sur le monde étant d'entrée de jeu traversé, annoncé, donné par la parole de l'autre. De surcroit, cet effet de citation est polyphonique puisqu'il condense le Balbec brumeux, funéraire et antique de Legrandin avec le Balbec de Swann, dont l'église est d'un curieux gothique presque persan, donnant à rêver le Balbec-Nature aussi comme œuvre d'art. Télescopage des récits ou des visions qui fait de Balbec un vaste tableau d'Histoire tout autant qu'un sublime morceau de Nature (à quoi s'ajoute la vision tempétueuse du Narrateur); le Narrateur absorbant le monde - Nature et Histoire - en un seul tableau.

Le paragraphe qui suit cet élan poétique est celui, on oserait dire plus prosaïque, de la rêverie sur «les réclames des Compagnies de chemin de fer":

J'aurais voulu prendre dès le lendemain le beau train généreux d'une heure vingt-deux dont je ne pouvais jamais sans que mon cour palpitât lire, dans les réclames des Compagnies de chemin de fer, dans les annonces de voyages circulaires, l'heure de départ: elle me semblait inciser à un point précis de l'après-midi une savoureuse entaille, une marque mystérieuse à partir de laquelle les heures déviées conduisaient bien encore au soir, au matin du lendemain, mais qu'on verrait, au lieu de Paris, dans l'une de ces villes par où le train passe et entre lesquelles il nous permettrait de choisir; car il s'arrêtait à Bayeux, à Coutances, à Vitré, à Questembert, à Pontorson, à Balbec, à Lannion, à Lamballe, à Benodet, à Pont-Aven, à Quimperlé, et s'avançait magnifiquement surchargé de noms qu'il m'offrait et entre lesquels je ne savais lequel j'aurais préféré, par impossibilité d'en sacrifier aucun. (378-79)

Le guide horaire du train est pour ainsi dire un autre premier livre (tel François le Champi); livre du nom, des noms enchaînés les uns aux autres par la magie d'un chemin - d'une ligne - qui semble tracer un ordre (un sens) au-delà de la confusion première du monde. L'énumération, la série, apparaît là comme le degré zéro de l'écriture, l'ordre premier ou la séquence minimale du récit. Ce guide horaire arraisonne en effet la rêverie à un ordre précis en ce qu'il suppose un sujet capable de s'orienter dans l'espace et le temps. On peut d'ailleurs lire ce "point précis», cette "savoureuse entaille», cette «marque mystérieuse» comme le nécessaire repérage du sujet à l'égard d'une vision soumise à un continuel processus de métaphorisation et de métamorphose. Même s'il est support de la rêverie sur les noms - avec ce que cela suppose d'arbitraire et d'associations subjectives -, le guide horaire n'en reste pas moins le récit minimal et premier qui assure l'ancrage du sujet dans le temps et l'espace; déictique ou effet de coupure où se discernent le même et l'autre, l'ici et le là-bas, l'hier, le maintenant et le demain. Premier temps du sujet qui se confond dès lors avec l'énonciation des déictiques. Le train, symbole de ponctualité et de régularité - et, donc, de quasi-certitude -, évoque l'avènement du discernement et de son sujet; appel au réel encore, à l'extériorité, à l'arbitraire de son ordre qui dépasse toutes volontés. Cette scène s'articule également à la précédente (celle du sublime tableau de Balbec) par son appel au réel, à l'extériorité, même si la rêverie reprend de plus belle dès lors que le nom - Bayeux, Coutances, Quimperlé- relance le sujet-du-rêve (ainsi qu'on va le voir plus loin). 
La suite de ce passage nous montre enfin la souveraine plasticité de l'imagination du Narrateur qui, selon qu'il évoque Balbec ou le nord de l'Italie (Florence), passe d'un rêve à un autre, d'une modulation particulière de sa sensibilité à une autre soit du Balbec tempétueux et gothique au printemps parfumé et diapré de Florence: «Puis il arriva qu’une simple variation atmosphérique suffit à provoquer en moi cette modulation sans qu'il eût besoin d'attendre le retour d'une saison» (379). Le trésor d'images est donc d'abord celui qui lui vient du corps; entrelacement de sensations tout à la fois précises, diffuses et confuses à l'égard du monde qui l'entoure. Le Narrateur est ainsi, en son commencement, ce corps de souvenirs enchevêtrés - nœuds de sensations, d'images, et de signifiants - plutôt que l'idéale conscience de soi affranchie du sensible. En cela, il ne maîtrise pas encore sa rêverie - maîtrise qui ne saurait qu'être toute relative d'ailleurs -, ni le cours impétueux et intempestif de son imagination dans les eaux tumultueuses des sensations. Désir de maitrise qui s'affirme cependant dans la suite du texte:

Mais bientôt comme ces phénomènes naturels dont notre confort ou notre santé ne peuvent tirer qu'un bénéfice accidentel et assez mince jusqu'au jour où la science s'empare d'eux, et les produisant à volonté, remet en nos mains la possibilité de leur apparition, soustraite à la tutelle et dispensée de l'agrément du hasard [...]. Je n'eus besoin pour les faire renaître que de prononcer ces noms: Balbec, Venise, Florence, dans l'intérieur desquels avait fini par s'accumuler le désir que m'avaient inspiré les lieux qu'ils désignaient. (379-80)

Science de la magique appellation-épellation des noms depuis laquelle s'affirme la souveraineté de l'imagination du Narrateur, dont il cherche cependant à analyser l'enchantement qu'elle exerce sur lui; l'écriture s'avérant dès lors un patient travail d'exorcisme.

\section{NOMS MONOCHROMES}

(ou la jouissance première des signifiants)

Le Narrateur analyse ainsi comment lui-même passe de la passivité à la maîtrise - quand bien même ce serait pour déchanter, plus tard, de cette autre forme de rêverie. Car, parmi ces «lois propres» qui déterminent son rapport à la nomination, il y a d'abord celle où s'impose une vision monochrome:

Mais les noms présentent des personnes [...] une image confuse qui tire d'eux, de leur sonorité éclatante ou sombre, la couleur dont elle est peinte uniformément comme une de ces affiches, entièrement bleues ou entièrement rouges, dans lesquelles, à cause des limites du procédé employé ou par un caprice $d u$ décorateur, sont bleus ou rouges, non seulement le ciel et la mer, mais les barques, l'église, les passants. (380-81)

Processus d'uniformisation qui noue ensemble les divers objets que perçoit le sujet, lequel correspond en effet à un moment déterminé de la connaissance qu'il a alors du monde et de son propre désir; délimitation qui a pour effet de rendre sa vision du monde monochrome, excluant l'épaisseur de l'expérience ou l'altérité des choses et des êtres. Or si le nom est réceptacle du rêve, agglutination de sensations et d'associations diverses, il n'en demeure pas moins délimité par le désir. En cela, cette limitation est une première façon de fixer l'objet, de l'assigner à un sens; première forme de l'unité ou de l'unification qui, tout illusoire ou subjective qu'elle soit, permet au sujet de rêver les objets du monde dans l'horizon de son désir, comme un geste d'appropriation; geste premier et fondateur dont il lui faudra ensuite reconnaittre le leurre. Sans doute en est-il d'ailleurs toujours ainsi, alors que l'on peut considérer que le premier âge du signifiant (ou du nom) est celui où il le voit se confondre entièrement avec sa jouissance. La monochromie du nom équivaut ainsi à ce geste premier d'appropriation, lequel se heurte cependant au temps second de l'expérience.

Cette vision monochrome est donc - si l'on prend en considération ce qu'il en est de la nature même du sujet - à la fois vraie et fausse; vraie, dans la mesure où le sujet ne peut que reconfigurer en un foyer unifiant son expérience flottante du monde; fausse, puisque le poids de l'altérité défait ou corrige cette vision dès lors qu'il noue sa fantasmagorie à l'expérience toujours contingente qu'il fait du monde. Double temps du 
sujet qui oscille entre l'unification et la dispersion, laquelle scande toute son expérience perceptuelle et cognitive. Cette vision monochrome correspond ainsi à ce premier temps où le sujet n'a pu advenir qu'à s'inscrire dans ce double geste où il est à la fois nommé (premiers signifiants de son désir) et nommant, tentant aussi de maîtriser un tant soit peu le manque qui traverse toute parole; scène originaire où l'infans cherche à retrouver la plénitude du nom (et) de la jouissance ${ }^{5}$ ? La métaphore de la monochromie se laisse ainsi déchiffrer dans l'économie du signifiant comme le premier nom de la jouissance, nœud de corps, d'être et d'altérité.

Le nom est ainsi le foyer d'une multitude d'images reconfigurée selon le désir du Narrateur - du rêveur. Mais, on l'a vu, ce foyer est monochrome dans la mesure où il se construit sur un désir qui méconnaît une certaine expérience de l'altérité. Il y a d'ailleurs dans cette monochromie quelque chose qui relève du solipsisme puisque le Narrateur, évoquant les noms, réécrit, semble-t-il, un nouveau dictionnaire du monde, lequel n'est aussi que son propre roman; évocation enchanteresse où le signifiant semble précéder le signifié:

Le nom de Parme, une des villes où je désirais le plus aller, depuis que j'avais lu La Chartreuse, m'apparaissant compact, lisse, mauve et doux, si on me parlait d'une maison quelconque de Parme dans laquelle je serais reçu, on me causait le plaisir de penser que j'habiterais une demeure lisse, compacte, mauve et douce, qui n'avait de rapport avec les demeures d'aucune ville d'Italie puisque je l'imaginais seulement à l'aide de cette syllabe lourde du nom de Parme, où ne circule aucun air, et de tout ce que je lui avais fait absorber de douceur stendhalienne et $d u$ reflet des violettes. (380-81)

Nommer a quelque chose de magique, faisant apparaitre un monde délimité par le désir toutpuissant du sujet. Il suffit de prononcer les syllabes de quelque nom - formule incantatoire - pour donner à voir un tableau ou voir se déployer l'enchaînement des sensations et des récits qu'il renferme. Tel un coffret précieux rempli de prédicats qui, lorsqu'il est proféré, répand son trésor, le nom est comme le premier maillon d'une chaine à quoi se rattache un surnom (un éponyme), une périphrase, un récit. Cette vision monochrome du monde est aussi une autre façon de rappeler que le sujet est inscrit dans les signifiants premiers et secrets où son désir s'est noué. Sans doute, il ne suffit pas de constater le leurre de ce cratylisme qui traverse le texte proustien - et l'histoire de la littérature -; peut-être nous faut-il plutôt remarquer que cette rêverie motivante sur les noms est aussi un moment constitutif dans la mesure où le désir de cette motivation correspond au désir de fusion archaïque avec le monde (la Mère), là où règne supposément quelque idéal chant de béatitude. Cette pensée magique (ou monochrome) est d'ailleurs évoquée en rapport avec les "œuvres des primitifs" (383); en cela, langue rêvée de l'origine. Au commencement serait donc cet enchantement pour la musique du nom, pleine de ses associations subjectives, laquelle correspond aussi à quelque langue première de la jouissance. Ce rapport au nom relève enfin de la pensée magique dans la mesure où le sujet tend ici à nier l'arbitraire qui fonde la langue, se liant aux noms sur le mode d'une rêverie motivante ${ }^{6}$.

Au commencement règne ainsi cette loi d'association subjective, stratifiée et rhizomatique, plaçant l'avènement de la conscience dans l'aprèscoup d'une parole qui, toute fragmentaire, fragile et provisoire qu'elle soit, cherche à dénouer cette fascination originaire pour le signifiant, c'est-à-dire pour cette lettre-corps qui marque l'entrée du sujet dans l'ordre du désir. Logique du temps premier où le nom apparaît d'abord comme un agrégat de désirs (de signifiants) qu'avec l'expérience, cependant, le Narrateur saura ou voudra discerner, rompant ainsi avec le plaisir parfois ambigu de cette profération. Cette magique appellation-épellation n'a-t-elle pas d'ailleurs quelque chose de vaguement onaniste puisque le sujet ne (se) nomme ici que pour se donner immédiatement - et dans une sorte d'hallucination l'objet de son plaisir? Plaisir des noms qui est comme le corps premier de l'auto-érotisme. Plaisir des noms (Florence, Venise, Pise) qui est décrit aussi comme le 
ressassement insatiable d'une "mélodie» (383), vécu comme «quelque chose d'aussi profondément individuel que si ç'avait été un amour, un amour pour une personne [...]» (384). Ressassement amoureux qui ne passe pas par l'expérience d'une rencontre tangible, mais plutôt par l'évocation obsessionnelle et fantasmatique de quelques noms: "Ces images irréelles, fixes, toujours pareilles, remplissant mes nuits et mes jours [...]» (383). Ruminations qui piègent le Narrateur dans une monomanie solipsiste, prisonnier malheureux du même réseau d'images: "Peut-être même la simplification de ces images futelle une des causes de l'empire qu'elles prirent sur moi " (382). Ce jouissif ressassement du nom s'avère donc aussi ravissement dysphorique ou fascination mortifère puisque le Narrateur est pour finir dépossédé, bien qu'il croit par moments être celui qui en maitrise la scène. D'où l'ambiguité d'une telle posture où le Narrateur est à la fois tout-puissant et, cependant, enfermé dans le rêve de ses associations subjectives. On peut également reconnaître, dans cette rêverie sur le nom, le geste d'un sujet jouant du nom comme l'enfant de la bobine, le faisant apparaître et disparaître à volonté; Fort-Da, ou scansion première où se donnent à symboliser la présence ET l'absence de l'autre; jeu qui permet justement de symboliser ce rapport à l'autre sur le double mode de la maîtrise ET du dessaisissement ${ }^{7}$. Comme cette bobine, le nom serait ce avec quoi le Narrateur s'amuse et expériemente son désir, tenant à distance notamment le piège séduisant, mais étouffant, d'une imagination absolument souveraine.

\section{DÉSINCARNATION}

Cet épisode sur les noms se termine par l'annonce tant attendue du voyage à Florence et Venise, sonnant en quelque sorte la fin de la rêverie du Narrateur qui réagit alors ainsi: «[...] je sentis s'opérer en moi une miraculeuse désincarnation; elle se doubla aussitôt de la vague envie de vomir [...]» (386). L'annonce du voyage apparaît comme un événement trop lourd à porter, inassumable peut-être parce qu'il montre l'incommensurable écart entre le rêve et la réalité.
N'est-ce pas le corps qui se dresse là, paniqué devant cette épreuve redoutée? «Miraculeuse désincarnation» d'un corps qui se défait soudainement de son corps-derêveries pour se soumettre à celui de la souffrance, du manque et de la finitude; comme si, au moment de franchir la ligne entre la souveraineté du rêve et l'expérience de l'autre (dans ce cas, de la ville), une nausée s'emparait de lui pour lui faire "vomir» les noms idéalisés, recrachant son rêve pour ne laisser là qu'un malade du rêve, incertain du rapport qui prévaut entre lui-même, les mots et les choses. Comme si le corps le rappelait à l'ordre, lui rappelait que le corps a son ordre dans la mesure où il y a renoncement à la toute-puissance des idées, sinon à la magie $^{8}$. La "désincarnation» s'avère ainsi comme le renoncement à ce corps souverain de l'imagination, à la fois tout-puissant et fragile, pour mieux renaître dans un autre corps, et advenir, à travers cette épreuve, comme un autre; moment de vérité qui s'affirme justement par l'assomption de la distance entre les mots et les choses, et la reconnaissance de l'altérité:

Ainsi elle [Venise] et la Cité des lys n'étaient pas seulement des tableaux fictifs qu'on mettait à volonté devant son imagination, mais existaient à une certaine distance de Paris qu'il fallait absolument franchir si l'on voulait les voir, à une certaine place déterminée de la terre, et à aucune autre, en un mot étaient bien réelles. (385)

Franchir une quelconque distance entre deux points de l'espace suppose donc, contrairement à la volatilité de l'imagination, un autre corps soumis aux contingences du temps et de l'espace. Ce qui s'oppose ici à l'expérience vertigineuse de la rêverie - laquelle suppose toutes sortes de vitesses et d'amalgames que le jeu de la métaphore et de la métonymie proustiennes donne à voir - est l'affirmation d'un autre espacetemps dominé par le point - «à une certaine place déterminée de la terre» - et la ligne, le tracé de la distance, aussi bien dire, par la géométrie. Le texte s'inscrit ainsi dans un double procès où se remarquent les deux temps, inextricablement noués, du sujet: souveraineté de l'imagination et contingence de l'espace-temps. Double procès qu'assume enfin le 
Narrateur: «[...] les deux Cités Reines dont j'allais avoir, par la plus émouvante des géométries, à inscrire les dômes et les tours dans le plan de ma propre vie" (385). La «plus émouvante des géométries» réside en effet dans la superposition, la double focalisation de ces deux plans - subjective et objective - qui traverse le sujet, en même temps.

Il n'y a donc pas à choisir entre l'un et l'autre pour vanter l'impossible constitution d'un sujet ou, au contraire, affirmer l'existence du seul sujet de la métaphysique de la présence et de l'identité -, puisqu'il s'agit plutôt de reconnaitre ce double foyer comme étant au fondement de l'expérience du monde, alors que le sujet s'avère à la fois rêveur et arpenteur géomètre. C'est sans doute en cela que la fiction proustienne se laisse également lire à ce niveau comme un vaste traité sur le bon usage de l'imagination.

GILBERTE! (le deuxième temps du nom)

L'épisode qui suit celui de la rêverie sur les noms concerne la rencontre entre le jeune Narrateur et Gilberte, fille d'Odette et de Charles Swann. Une autre expérience du nom s'impose ici qui implique justement la reconnaissance de l'autre. L'épreuve du désir, de l'amour, de la jalousie et de la souffrance bref, du manque et de la non-maîtrise - suppose en effet un nouveau rapport au nom. Véritable coup de cymbale dans le rêve (dans le texte), le Narrateur s'éveille enfin ici tout autrement à l'écoute du nom:

Ce nom de Gilberte passa près de moi, évoquant d'autant plus l'existence de celle qu'il désignait qu'il ne la nommait pas seulement comme un absent dont on parle, mais l'interpellait; il passa ainsi près de moi, en action pour ainsi dire, avec une puissance qu'accroissait la courbe de son jet et l'approche de son but [...]. (387)

L'interpellation en ce qu'elle suppose tout le registre de la demande - appel, déclaration, promesse, prière - donne à entendre la dimension intersubjective de la parole: «[...] il ne la nommait pas seulement comme un absent dont on parle, mais l'interpellait [...]» (387). Scène qui laisse le Narrateur d'abord douloureusement exclu, montrant bien que la gratification du babil magico-imaginaire sur les noms ne lui est plus d'aucun secours. L'expérience de l'autre commence ainsi par celle de la douleur: «[...] toute cette vie séparée de la mienne que par deux fois, condensée dans le nom de Gilberte, j'avais sentie passer si douloureusement près de moi [...]» (388). Un nom est lancé - une force, une action, un désir - par un autre et pour un autre, lequel est le contraire du nom de la rêverie (nom monochrome du ressassement, complaisante mélodie, ritournelle, litanie qui mime le règne des signifiants premiers de la jouissance). Le nom est également ici parcours, destination, ou chemin par «la courbe de son jet et l'approche de son but»; il trace ainsi une ligne entre deux êtres, dessinant les lignes congruentes d'une autre géométrie du désir. Si l'interpellation rompt avec la rêverie des signifiants, le nom oblige ainsi le sujet à faire l'épreuve d'une distance, d'une séparation, d'une coupure; violence qui arrache le Narrateur à son corps-de-rêve et qui a d'ailleurs la violence du cri :

[...] et le jetait en plein air comme un cri; - laissant déjà flotter

dans l'air l'émanation délicieuse qu'il avait fait se dégager, en les touchant avec précision, de quelques points invisibles de la vie de Mlle Swann [...] formant, passager céleste au milieu des enfants et des bonnes, un petit nuage d'une couleur précieuse, pareil à celui qui, bombé au-dessus d'un beau jardin du Poussin, reflète minutieusement, comme un nuage d'opéra plein de chevaux et de chars, quelque apparition de la vie des dieux; - jetant enfin, sur cette herbe pelée, à l'endroit où elle était un morceau à la fois de pelouse flétrie et un moment de l'après-midi de la blonde joueuse de volant [...] une petite bande merveilleuse et couleur d'héliotrope, impalpable comme un reflet et superposée comme un tapis, sur lequel je ne pus me lasser de promener mes pas attardés, nostalgiques et profanateurs [...]. (387)

Ce cri - Gilberte! - interrompt la rêverie du Narrateur, comme si l'altérité se manifestait à lui par cette violence première; épreuve de la contingence contre le babil solipsiste. Le nom, lancé avec force, éclat, résonne pour éveiller au désir; ce cri marquant l'avènement d'une inscription ou d'un repère, laissant d'ailleurs dans l'air une trace précise «de quelques points invisibles de la vie de Mlle Swann». L'appel au 
précieux et au minutieux suggère une expérience du dicible, sinon de la précision, qui contraste avec le vertige impressionniste où baigne, à d'autres moments, le Narrateur. Ce nom, pareil à un petit nuage qui se détache du décor, se laisse en effet admirer comme un tableau; ce qui suppose ici un renversement poétique où le nuageux de la perception impressionniste est pour ainsi dire retraduit ou reconfiguré selon la ligne discernable d'un désir. La vision du nuage - paradigme d'un certain flou impressionniste - se singularise, dessinant le paysage de cet amour, lisible enfin comme peut l'être le nom même de Gilberte. De même, ce nom fait saillie sur le gazon, en laissant «une petite bande merveilleuse et couleur d'héliotrope, impalpable»; il s'incarne ainsi, corps de lumière qu'on ne peut toucher mais qui est malgré tout la trace d'un désir reconnu. "Gilberte!» vient donc interrompre, ponctuellement, le registre impressionniste de la perception qui menace de dissoudre le sujet dans l'indifférencié; nom de l'amour (d'un désir) qui s'incarne enfin à la surface toujours labile des sensations et des perceptions.

Cette rencontre avec Gilberte, si elle est d'abord réveil et ravissement, est aussi cependant expérience de la séparation, plaçant le Narrateur dans l'attente d'une autre rencontre. D'ailleurs, l'espoir d'une telle rencontre fait de lui un nouvel interprète de la nature, recadrant sa vision autour de son désir (jusqu'à la fascination, encore); ainsi en est-il de ce rayon de lumière dont il observe le jeu sur le balcon et qui s'avère, en même temps, promesse de beau temps et de l'éventuelle présence de Gilberte aux ChampsÉlysées:

Lierre instantané, flore pariétaire et fugitive! [...] de celles qui peuvent ramper sur le mur ou décorer la croisée; pour moi, de toutes la plus chère depuis le jour où elle était apparue sur notre balcon, comme l'ombre même de la présence de Gilberte [...]. (389)

Ce rayon de soleil qui se répand sur le monde, découpant le balcon, métaphore de Gilberte, s'offre ainsi à déchiffrer comme étant l'autre nom de son désir; il est le nom (Gilberte-lierre) qui, telle une fleur, s'agrippe, grimpe, recouvre, enveloppe un autre corps. «Gilberte» devient ainsi le nom paradigmatique du nouage, de l'alliance, du désir et de l'amour; nomfleur, nom de l'efflorescence amoureuse qui se lie à l'autre. Et ce jeu de lumière sur le balcon, scène de l'attente, se donne justement à lire selon une autre esthétique que celle d'un certain fondu impressionniste:

[...] je la voyais atteindre à cet or inaltérable et fixe des beaux jours, sur lequel l'ombre découpée de l'appui ouvragé de la balustrade se détachait en noir comme une végétation capricieuse, avec une ténuité dans la délinéation des moindres détails qui semblait trahir une conscience appliquée, une satisfaction d'artiste [...]. (389)

Le jeu de la lumière n'est donc pas ici (con)fusion des objets puisque le rayon donne plutôt à voir le monde dans sa discernabilité ("avec une ténuité dans la délinéation des moindres détails»), sinon, même, dans sa fixité ( je la voyais atteindre à cet or inaltérable et fixe»). La description du balcon est donc moins impressionniste que géométrisante; travail d'écriture où se donnent à lire et à désirer des corps distincts. L'attente du Narrateur, son désir de l'autre, se soutient donc d'une autre scène imaginaire que celle de la rêverie monochrome, dès lors que la vision géométrisante s'avère travail de discernement et de reconnaissance. Le rayon découpe, sculpte, discerne comme le Narrateur discerne dans les signes, le jeu de la lumière, la présence d'un autre signe, celui de la possible rencontre avec Gilberte. Travail de discernement et d'analyse où le Narrateur n'est pas simplement absorbé par la danse des corps dans la lumière chatoyante, mais se repère dans le monde; comme si le désir était tout autant expérience de l'illusion - qui est l'autre? - que de la lucidité. La géométrisation des images soutient donc le Narrateur dans sa quête de discernement et de lucidité, tel un art de la fine broderie, sinon de l'écriture: «[...] le soleil apparu entrelaçait des fils d'or et brodait des reflets noirs" (390). Contemplation de la lumière qui suppose l'aigu, le tranchant, la pointe du regard; expérience qui est moins celle de ces masses 
indistinctes fondant sur les objets que celle de la délimitation, de la séparation, de la ligne, de l'analyse. On remarque ainsi un double processus du rayonnement solaire puisqu'il baigne (regard impressionniste) et découpe (regard géométrisant et cubiste). Double regard que le texte proustien enroule et déplie tout à la fois autour de sa quête de l'autre et du monde.

Le texte se déploie en effet comme une nappe faite d'une double trame où se dévoilent tout à la fois le flou et le tranchant d'un certain regard. Cette attente langoureuse de Gilberte, mobilisant chez le Narrateur le travail de l'interprétation, a d'ailleurs d'autres objets ou d'autres scènes: «Tout à coup l'air se déchira: entre le guignol et le cirque, à l'horizon embelli, sur le ciel entr'ouvert, je venais d'apercevoir, comme un signe fabuleux, le plumet bleu de Mademoiselle» (391). «Déchirure» dans la toile bariolée des perceptions (l'immobilité glacée des Champs-Élysées en hiver), laquelle fait l'événement, fait signe, pose le singulier, autant dire la marque, le trait, le nom de l'Aimée. On retrouve enfin la même image quelques pages plus loin, alors que le texte tresse ses divers motifs:

[...] cette attente finissait par me rendre plus émouvants, non seulement les Champs-Élysées entiers et toute la durée de l'aprèsmidi, comme une immense étendue d'espace et de temps sur chacun des points et à chacun des moments de laquelle il était possible qu'apparût l'image de Gilberte [....]. (398)

Comme si l'espace-temps infini du monde (et l'impression perceptuelle de la dissolution-osmose des corps) se stabilisait enfin dans le nom de Gilberte, foyer d'un désir donnant sens et consistance à «l'immense étendue» de l'expérience. Le nom s'avère ainsi point de repère dans le monde des sensations multiples et infinies; ponctuation qui scande le flux ininterrompu des perceptions, des illusions, des savoirs.

Ce regard qui discerne n'est pourtant qu'un moment dans la conscience toujours en mouvement du Narrateur qui conserve aussi une image évanescente et instable de Gilberte (comme ce sera le cas aussi pour Albertine, et son fameux grain de beauté). Mais cette image n'est pas nécessairement perdue à jamais, étant plutôt toujours à refaire:

[...] si depuis la veille je portais dans ma mémoire deux yeux de feu dans des joues pleines et brillantes, la figure de Gilberte m'offrait maintenant avec insistance quelque chose que précisément je ne m'étais pas rappelé, un certain effilement aigu du nez [...]. (394)

La figure (l'image) se recompose ainsi autour d'un trait, d'une marque ou d'un signe qui la rend à nouveau reconnaissable. Le souvenir imagé de l'autre peut bouger, s'effacer, se perdre; mais toute rencontre est aussi l'occasion d'une nouvelle «mise au point». Le Narrateur prend là encore conscience de la division qui le détermine, reconnaissant en lui deux «Moi»; l'un, hanté par l'incertitude des images, des souvenirs, et un autre, reconnaissant le même (Gilberte) à travers le changement: «[...] et que venait de me faire saluer et reconnaitre un instinct aveugle [...] aussitôt tout se passait comme si elle et la fillette qui était l'objet de mes rêves avaient été deux êtres différents" (394). Ainsi, et malgré le subjectivisme de toute perception, le Narrateur ne se vautre pas sur l'image, idéale et idéalement fixe de Gilberte; au contraire, la rencontre de la jeune fille - et l'événement toujours répété de la contingence qu' elle incarne - le contraint à réviser son jugement ou sa perception. Travail continuel de reconfiguration où alternent l'illusion et la lucidité.

À cet égard, le texte proustien ne cesse de mettre en scène cette loi où le premier temps de toute rencontre n'advient aussi qu'après coup. Premier temps qui ne se saisit que dans la répétition où est posé l'avènement toujours déjà différé d'une identité ou d'un «présent» toujours ponctuel.

\section{ÉCRIRE SON NOM EN VAIN}

Cette épreuve de l'altérité est lisible également lorsque le Narrateur, écrivant sans cesse le nom de l'Aimée, comprend qu'il ne fait là que se vautrer dans l'impuissance dès lors que la magique appellationépellation s'épuise et ne tient pas la promesse de quelque incarnation fabuleuse. Qu'est-ce en effet que jouir du nom de l'autre s'il ne s'offre pas de lui-même à désirer? 
Certes, à toutes les pages de mes cahiers, j'écrivais indéfiniment son nom et son adresse, mais à la vue de ces vagues lignes que je traçais sans qu'elle pensât pour cela à moi, [...] je me sentais découragé parce qu'elles ne me parlaient pas de Gilberte qui ne les verrait même pas, mais de mon propre désir qu'elles semblaient me montrer comme quelque chose de purement personnel, d'irréel, de fastidieux et d'impuissant. (393)

Nom où s'incarne la pure promesse d'une rencontre, d'un avenir, d'une possession; travail d'écriture cependant véhément, en dépit de cette prière incantatoire du nom de l'Aimée où apparaît la croyance originaire dans les pouvoirs de la nomination ${ }^{9}$. Comme si le nom de l'Aimée (de l'amour) saturait, en vertu de l'aura merveilleuse du désir, les aléas d'une vie sentimentale où s'expérimente le Narrateur et sur laquelle toutefois l'écriture romanesque tisse nécessairement sa toile. En cela, ce livre-cahier est surtout celui de l'impuissance, de la vide et vague rêverie autour d'un nom qui ne se laisse pas posséder. Voilà pourquoi ce livre est aussi celui de l'expérience du manque à partir duquel est symbolisée la place de l'autre; deuil, là encore, à l'égard de la rêverie incantatoire alors que la première jouissance pour le nom cède la place à la reconnaissance d'une altérité immaîtrisable. Mais le livre apparemment vain du ressassement et de l'appel est pour ainsi dire la première phrase à venir, sinon le premier récit, de ce qui ne sait pas encore se dire. Ce nom qui ne cesse de s'écrire à la pointe d'un désir véhément est donc là encore le livre premier, et $L a$ Recherche ne s'écrit-elle pas sur la toile de ce livre premier, palimpseste d'un cahier d'écolier qui tout à la fois dévoile et retisse autrement le nom d'un amour ancien? Le livre premier est livre de l'adoration, gloire du nom de l'Aimé(e) qui apparemment contient tous les livres secrets du Narrateur, le nom glorieux de l'amour valant, semble-t-il, tous les récits passés, présents et à venir ${ }^{10}$.

Cette rencontre avec Gilberte est donc l'autre scène et l'autre économie subjective du nom. Sur cette scène se déploie aussi, on l'a vu, un autre discours imaginaire où, s'opposant à l'esthétique du flou, le motif géométrique s'impose pour fixer le sujet dans la trame d'une expérience de l'altérité. À cet égard, la bille d'agate que le Narrateur offre à Gilberte a sans doute valeur de symbole:

Je regardais avec admiration, lumineuses et captives dans une sébile isolée, les billes d'agate qui me semblaient précieuses parce qu'elles étaient souriantes et blondes comme des jeunes filles et parce qu'elles coûtaient cinquante centimes pièce. [...] Elles avaient la transparence et le fondu de la vie. [...] J'aurais aimé qu'elle pût les acheter, les délivrer toutes. (395)

Cette formule, «la transparence et le fondu de la vie», on peut justement la traduire comme étant l'expression des deux temps, inextricablement noués, du sujet: le «fondu» étant caractéristique d'une expérience où prédomine l'imaginaire impressionniste des magmas (miroir d'un sujet qui éprouve le monde sur le mode de l'indistinct), alors que la «transparence» relève davantage d'une expérience où s'affirme l'imaginaire consistant de la ligne et de la géométrie, transparence du cristal et figure de l'esprit lucide en quête de sa vérité - en tant qu'il désire ou qu'il cherche à recueillir «un peu de temps à l'état pur» ${ }^{11}$. Cette bille se laisse ainsi déchiffrer comme la figure même du récit ou de la poétique proustienne. De plus, elle symbolise le lien à l'autre puisqu'elle fournit l'occasion d'une rencontre et d'un échange entre le Narrateur et Gilberte où entre en jeu la reconnaissance de l'autre, c'est-à-dire le nouage de deux paroles autour des gestes de payer, de donner et de conserver:

Pourtant je lui en désignai une qui avait la couleur de ses yeux.
Gilberte la prit, chercha son rayon doré, la caressa, paya sa
rançon, mais aussitôt me remit sa captive en me disant: «Tenez,
elle est à vous, je vous la donne, gardez-la comme souvenir». (395)

L'objet noue le sujet à l'autre, en tient lieu, "permane» dans la suite du temps pour se faire histoire. Tel un symbole (un sumbolon) - cet objet que les anciens brisaient en deux pour en conserver chacun une partie et mieux se reconnaître par la suite -, cette bille les unit et leur sert de repère ou de mot de passe ${ }^{12}$. Le «rayon doré», la bille d'agate, tranche ainsi 
dans le magma de la rêverie amoureuse pour nouer ensemble deux paroles.

La scène qui suit est d'ailleurs tout aussi symbolique de cette reconnaissance de l'altérité, scène d'écriture où il s'agit de passer par l'autre et la loi:

Le lendemain elle m'apporta, dans un paquet noué de faveurs mauves et scellé de cire blanche, la brochure qu'elle avait fait chercher. [...] j'eus peine à reconnaître les lignes vaines et solitaires de mon écriture sous les cercles imprimés qu'y avait apposés la poste, sous les inscriptions qu'y avait ajoutées au crayon un des facteurs, signes de réalisation effective, cachets $d u$ monde extérieur, violettes ceintures symboliques de la vie, qui pour la première fois venaient épouser, maintenir, relever, réjouir mon rêve. (395-96)

Cette brochure est un commentaire de Bergotte sur Racine; mais elle est surtout l'occasion d'une scène d'écriture où apparaît la dimension symbolique de la reconnaissance de l'autre, du nom. La lettre - le télégramme, le petit bleu - doit en effet, pour arriver à son destinataire, être oblitérée par l'autre: le maitre de poste qui atteste par ses initiales du respect de l'affranchissement et, possiblement, de la bonne adresse. Un tiers s'interpose ainsi, interrompant la circulation de la lettre pour mieux en valider le cours; il est en quelque sorte le représentant de la loi, celui qui assure la circulation de la lettre et, dans une certaine mesure, celle des noms. L'altérité, l'extériorité du trajet ébranle ainsi le ressassement du nom adoré et place cette rêverie sous le regard d'un autre, paradigme de la loi. Le nom devient ainsi signature oblitérée - «violettes ceintures symboliques de la vie» - donnant, ponctuellement du moins, consistance et limite à la rêverie; comme si l'imagination retrouvait là le ceinturage de la loi, garde-fou de toute rêverie. Ainsi, et alors que la première rêverie sur les noms prenait la forme d'une rêverie monomane - magique syllabaire de l'auto-érotisme -, l'usage des noms lui échappe enfin dès lors que l'autre (Gilberte) le nomme. En le nommant, c'est d'ailleurs comme si elle le tenait dans sa bouche, "[...] moi-même, nu, sans plus aucune des modalités sociales [...]» (396). Le Narrateur n'est plus ici le souverain onomaturge de sa rêverie, mais, et parce qu'il est nommé, l'objet d'un autre qui le dessaisit un tant soit peu de lui-même.

Cette rencontre avec Gilberte est aussi l'occasion d'une nouvelle rencontre avec Charles Swann, lequel apparaît dès lors sous un autre éclairage:

[...] pour moi Swann était surtout son père, et non plus le Swann

de Combray; comme les idées sur lesquelles j'embranchais

maintenant son nom étaient différentes des idées dans le réseau

desquelles il était autrefois compris [...] il était devenu un

personnage nouveau; je le rattachai pourtant par une ligne

artificielle, secondaire et transversale à notre invité d'autrefois

[...]. (400)

Épreuve du temps encore qui, on le sait, altère l'image ou le souvenir. Pourtant, et telle une araignée, le Narrateur trace des lignes, établit des réseaux, lie le passé au présent. La ligne est «artificielle, secondaire et transversale», soit; mais elle tient, fait tenir ou rattache ce qui autrement ne serait que traces dispersées par le vent. C'est cette «ligne" qui noue ensemble le même et l'autre, donnant malgré tout consistance au capricieux processus de la perception. Cette ligne est en définitive celle-là même du récit, de l'histoire, de la mémoire; anastomose où s'inscrit l'avènement d'un sujet, mélange d'expériences vertigineuses et d'épiphaniques certitudes.

De même, le nom de Charles Swann lui paraît désormais «nouveau», étranger: «Il était toujours présent à ma pensée et pourtant elle ne pouvait pas s'habituer à lui. Je le décomposais, je l'épelais, son orthographe était pour moi une surprise» (405). Dans ce second temps du nom, celui-ci a aussi quelque chose d'immaîtrisable ou d'illisible, sans doute parce qu'il n'est pas le tout de l'identité, mais le point d'ancrage de cette identité à la fois reconnaissable et voilée dans le mystère de son origine. Mais pour saisir le mouvement de cette incertitude identitaire, ne fautil pas que se maintienne le nom, trace minimale et première de l'identité sans quoi le jeu du même et de l'autre serait dissous dans une complète altérité, s'avérant dès lors altération. S'il y a un sens à parler du devenir-autre du sujet, c'est dans la mesure où le nom est cette instance qui, d'entrée de jeu, le lie à son 
histoire et à la loi; sans quoi il n'y aurait pas de sujet, mais quelque chose justement d'innommable, en état de continuelle dissolution. En cela, le texte de Proust montre, simultanément, la fragilité de ce nouage du sujet à son nom (incertitude vertigineuse) et sa nécessité qui l'assujettit à la loi.

Double processus qui donne au texte et à la phrase cette forme tressée; double trame faite de vertiges et de certitudes là où alternent, sur le plan de l'imaginaire et de la constitution du sujet, le flou et la ligne, la fumée et le cristal, l'inconnu et la certitude ${ }^{13}$. La poétique du texte proustien est en cela pareille en effet à une robe (de Fortuny) - autre paradigme formel de la composition de la Recherche - en ce que cet art unit le chatoiement des tissus luxueux avec l'art du pli, c'est-à-dire de la ligne; la robe, le texte proustien, étant simultanément un art fait de volumes mouvants et changeants qu'accentue quelque séduisante démarche et que seul un art précis du pli permet de souligner ${ }^{14}$. Ce qui en d'autres lieux ne serait qu'une plate opposition s'avère ainsi chez Proust le subtil agencement de ce qui est à la fois multiple et, ponctuellement, singulier.

\section{L'OUVRIÈRE INCONNUE-INVISIBLE}

Le Narrateur est donc dans l'attente d'une lettre d'amour qui ne viendra jamais. Cette attente implique un rapport au temps et au savoir qui le fait bouger. De la rêverie sur les noms à l'épreuve du nom de l'autre (par l'amour, la souffrance), le Narrateur est en effet amené à reconnaître une division qui le traverse et qui est celle, en somme, du conscient et de l'inconscient. Ainsi, il a beau tisser, au fil des jours, le scénario rêvé de son amour pour Gilberte, il n'empêche qu'une autre loi est à l'œuvre qui retisse autrement la toile de cet amour:

Et tandis que mon amour, attendant sans cesse du lendemain l'aveu de celui de Gilberte, annulait, défaisait chaque soir le travail mal fait de la journée, dans l'ombre de moi-même une ouvrière inconnue ne laissait pas au rebut les fils arrachés et les disposait, sans souci de me plaire et de travailler à mon bonheur, dans un ordre différent qu'elle donnait à tous ses ouvrages. (403) [...] Il disait enfin, l'ordre nouveau dessiné par l'ouvrière invisible, que si nous pouvons désirer que les actions d'une personne qui nous a peinés jusqu'ici n'aient pas été sincères, il y a dans leur suite une clarté contre quoi notre désir ne peut rien et à laquelle, plutôt qu'à lui, nous devons demander quelles seront ses actions de demain. (404)

Le Narrateur amoureux s'avère ici divisé entre ce qu'il croit vouloir et l'évolution secrète de son désir. Rêvant amoureusement de Gilberte le jour, quelque chose se défait lentement, inconsciemment, dès lors qu'il est déçu, épuisé, et las de cet amour. Le désir évolue, (se) creuse (sur) une faille où apparaît un sujet toujours déjà divisé entre ce qu'il sait ou croit savoir de son désir; quête infinie avec ses vertiges et ses îlots de certitude. Tout se passe donc comme si le Narrateur était traversé par deux temps (et deux savoirs): un temps diurne où il persévère dans ses illusions (ses rêves) et un temps nocturne où il obéit ultimement à son corps, lequel révèle ce qu'il n'ose encore s'avouer. Temps secret qui pourra éventuellement s'exposer à la lumière du jour, s'inscrire dans le temps diurne pour devenir cette scène secrète enfin assumée. Division qui suppose une voix encryptée dans une autre, voix de ventriloque parlant de l'intérieur de la première pour mieux la trahir, l'égarer, la dévoiler. Voix du lapsus, voix secrète du désir; voix de l'inconscient. L'Ouvrièreinconnue-invisible est donc à l'écoute du sujet, reconfigurant ou retissant, telle une araignée, la mise en scène de son désir; processus qui n'abolit pas sa division, mais la lui donne à symboliser comme un continuel travail d'assomption. Le désir a donc son enchaînement, sa loi, sa scène de vérité sur laquelle il vient buter, et ce malgré toutes les parades où il se joue, s'explore ou se dévoile.

Cette «clarté contre quoi notre désir ne peut rien» est bien l'autre lucidité ou le second temps du savoir et de la clairvoyance. Cette désillusion a également pour effet de dissoudre l'amalgame enchanteur des noms, d'effacer la beauté des pages écrites de Bergotte sur Racine (cause de son amour pour Gilberte), ou de rompre le pouvoir magique de la bille d'agate:

[...] je m'apercevais qu'elles étaient antérieures à cet amour,

qu'elles ne lui ressemblaient pas, que leurs éléments avaient été 
fixés par le talent ou par les lois minéralogiques avant que

Gilberte ne me connût [...]. (403)

Travail de déliaison, d'analyse et de discernement. Car si le mode de pensée par association est premier, il n'est pas dit que le sujet ne puisse en dénouer le fil pour reconnaître le désir qui l'anime, en puisant dans une lucidité qui, paradoxalement, précède momentanément la conscience.

L'Ouvrière-inconnue-invisible est donc la figure qui incarne cette division originaire faite d'aveuglement et de lucidité qui structure le sujet. Or, le Narrateur ne peut que postuler la manifestation de cette lucidité, tout comme il ne peut que supposer que son expérience du monde n'est pas entièrement un abîme de folie. Ne touchons-nous pas là le noyau irréductible de toute démonstration alors que l'on bute sur un principe indémontrable, sauf à reconnaître quelque véracité ou bonté divine gardienne de la raison (comme Descartes)? Ce noyau originaire indémontrable se trouve en effet surmonté par l'invocation de quelque figure tutélaire, gardienne de l'avènement du sens. À cet égard, on peut dire que l'ouvrière apparait comme la personnification (vaguement chimérique) d'une origine qui ne se laisse justement anticiper que par quelque figure, et non par la supposée pureté spéculative du concept ${ }^{15}$.

À l'origine serait donc aussi la figure, borne délimitant la folie et la lucidité, donnant à symboliser une inquiétante altérité qui erre dans les parages de l'innommable. Le récit invente ici la figure qui garde le Narrateur de tomber dans le magma impressionniste des sensations, tout en donnant forme à cette expérience ${ }^{16}$.

\section{LE BON ANGE DE LA CERTITUDE}

Tout le début de la Recherche peut d'ailleurs être lu comme une autre variation sur le thème de l'illusion et de la lucidité. On se souviendra en effet que le texte s'ouvre sur une expérience où le Narrateur apparaît dans l'état incertain entre la veille et le sommeil, passant de la lecture - du rêve éveillé? - au rêve, lisant et rêvant le livre sur lequel il s'est endormi, ne sachant plus par moment s'il lit, rêve ou dort:
Longtemps, je me suis couché de bonne heure. Parfois, à peine ma bougie éteinte, mes yeux se fermaient si vite que je n'avais pas le temps de dire: "Je m'endors». Et, une demi-heure après, la pensée qu'il était temps de chercher le sommeil m'éveillait; je voulais poser le volume que je croyais avoir encore dans les mains et souffler ma lumière; je n'avais pas cessé en dormant de faire des réflexions sur ce que je venais de lire, mais ces réflexions avaient pris un tour particulier; il me semblait que j'étais moimême ce dont parlait l'ouvrage: une église, un quatuor, la rivalité de François Ier et de Charles-Quint. (3)

La Recherche s'ouvre ainsi, non sans ironie pourraiton dire, sur cette phrase impossible - «je m'endors»-, et sur cette expérience tout à la fois ordinaire et mystérieuse d'une certaine perte de conscience, prélude à l'aventure d'une autre dimension du savoir de soi. D'entrée de jeu, le lecteur est ainsi placé devant ce point impossible à cerner où l'état de veille se condense à celui du rêve, montrant la porosité, la fluidité et la plasticité de la conscience. La Recherche s'ouvre en effet sur ce vacillement du sujet où, tels des vases communicants, le conscient et l'inconscient s'entremêlent; expérience première d'une conscience qui, chaque nuit, perd ses repères ordinaires pour mieux communier avec les images qui l'habitent.

À cet égard, lire et rêver sont par moments un seul et même geste. La lecture n'est-elle pas d'ailleurs le geste emblématique de cette plasticité du sujet dans la mesure où lire, c'est d'abord imaginer et, en même temps, s'identifier aux êtres et aux choses. Notons que cette identification est ici maximale: église, musique, personnage historique; tout peut en effet s'anthropomorphiser et s'animer pour déjouer l'ordre contingent qui sépare les êtres et les choses. Or, que le sujet soit église ou quatuor, c'est non seulement poser le registre de l'identification comme fondamental, mais c'est surtout personnifier, faire vivre et donc, symboliquement, donner la parole à ce qui en principe ne parle pas. Geste que l'on connaît sous le nom de personnification, si l'on veut bien considérer que cette figure correspond à ce noyau de croyance ancien et persistant d'un monde entièrement et fondamentalement animé. En cela, la lecture peut 
aussi être considérée comme le geste paradigmatique qui caractérise la présence du sujet au monde, alors qu'en lisant il ne cesse de s'identifier à ce qu'il imagine, animant et personnifiant ce qui autrement ne serait que l'inquiétant mutisme des choses. Si la Recherche s'ouvre ainsi sur une scène de lecture, d'identification ou de personnification, c'est justement qu'elle est peut-être la scène première d'un sujet qui, d'un monde muet, fait un monde parlant ou nommé (ainsi qu'on peut le lire par exemple dans La Théogonie d'Hésiode).

Cette personnification du monde est lisible dans l'épisode inaugural où le Narrateur décrit les chambres de son enfance:

Je me rendormais, et parfois je n'avais plus que de courts réveils d'un instant, le temps d'entendre les craquements organiques des boiseries, d'ouvrir les yeux pour fixer le kaléidoscope de l'obscurité, de goûter grâce à une lueur momentanée de conscience le sommeil où étaient plongés les meubles, la chambre, le tout dont je n'étais qu'une petite partie et à l'insensibilité duquel j'allais vite m'unir. (4)

Tout dort et s'endort, les êtres et les choses, s'animant semble-t-il du même souffle de vie, à tel point que le dormeur semble à peine se différencier de ce paisible «Tout» qui est comme le premier temps rêvé de l'existence. Sentiment d'unité quasi utérin - avant l'étincelle de conscience qui l'en séparera irrémédiablement - qui apparaît comme le sentiment fugace de l'existence avant qu'elle n'accède à sa dimension proprement humaine:

[...] et quand je m'éveillais au milieu de la nuit, comme j'ignorais où je me trouvais, je ne savais même pas au premier instant qui j'étais; j'avais seulement dans sa simplicité première le sentiment de l'existence comme il peut frémir au fond d'un animal [...]. (5)

Les chambres de l'enfance s'offrent ainsi à lire comme un autre premier livre dès lors que les murs, animés notamment par la lanterne magique, sont encore ici les surfaces animées d'un livre:

[...] et, à l'instar des premiers architectes et maîtres verriers de

l'âge gothique, elle substituait à l'opacité des murs d'impalpables irisations, de surnaturelles apparitions multicolores, où des légendes étaient dépeintes comme dans un vitrail vacillant et momentané. [...] Le corps de Golo lui-même, d'une essence aussi surnaturelle que celui de sa monture, s'arrangeait de tout obstacle matériel, de tout objet gênant qu'il rencontrait en le prenant comme ossature et en se le rendant intérieur, fût-ce le bouton de la porte sur lequel s'adaptait aussitôt et surnageait invinciblement sa robe rouge [...]. (10)

Tout comme les murs-bibliothèques du Grand Hôtel de la Plage à Balbec, les murs de la chambre s'animent, racontant une page de l'Histoire de France, alors que la matérialité contingente de la pièce se laisse docilement traduire dans quelque fabuleux récit; comme si l'ordre du possible ou de l'imagination arraisonnait à sa vision celui de la contingence et du factuel. Vision quasi hallucinée qui suppose que la lecture (l'écriture) est d'emblée transfiguration du monde. Cette transfiguration n'est pas folle hallucination, mais l'ordinaire d'un sujet qui n'anticipe le monde que depuis l'imaginaire, c'est-àdire depuis l'identification. Cela dit, si le récit de l'épisode des chambres montre aussi le vertige qui saisit le Narrateur dans le cours de ces identifications où il communie, sinon fusionne, autant avec l'espace de la chambre qu'avec le récit qui s'y condense, il n'en reste pas moins bien ancré dans la lucidité. Mais comment la justifier? C'est ici qu'intervient, après la longue et célèbre phrase des sept chambres où est raconté un certain vertige, une autre figure tutélaire, gardienne du sujet:

Certes, j'étais bien éveillé maintenant, mon corps avait viré une dernière fois et le bon ange de la certitude avait tout arrêté autour de moi, m'avait couché sous mes couvertures, dans ma chambre, et avait mis approximativement à leur place dans l'obscurité ma commode, mon bureau, ma cheminée, la fenêtre sur la rue et les deux portes. (8)

«Le bon ange de la certitude» - figure charmante aussi de l'habitude - se dresse ici pour ramener le voyageur à bon port après quelque vertigineux voyage astral, métempsycose ou voyagerie de l'âme dans un espace-temps soumis aux lois fantaisistes de 
l'imagination. Tel un ange gardien, il veille sur le sujet pour qu'il puisse discerner entre le possible et le contingent. C'est donc une autre figure tutélaire qui apparaît là pour dénouer l'aporie première du sujetde-la-parole et nécessairement répondre de l'origine de la lucidité sans répondre par le don (ou le non-savoir). L'ange de la certitude, tout comme l'ouvrière inconnue, est ainsi toujours déjà au cœur du sujet pour l'empêcher de se perdre dans quelque multiplicité dissolvante ${ }^{17}$.

\section{LE BCEUF EN GELÉE ORIGINAIRE (épilogue)}

Si cette archéologie de la conscience qu'est aussi la Recherche nous montre cette dépossession originaire du sujet, elle n'en raconte pas moins, on l'a vu, l'avènement d'une écriture et d'un regard qui, sur cet abîme tourbillonnant de sentiments confus, édifie peu à peu la ligne de partage depuis laquelle parvient à se fonder, ponctuellement, une parole. À cet égard, le mouvement entier de la Recherche ne se laisse pas tant emporter par quelque vague procès de dissémination (des identités, des expériences, des désirs...) qu'il se recentre sur des îlots de certitudes ponctuelles sans lesquelles le Narrateur ne saurait trouver cette consistance minimale où prendre appui pour connaître un tant soit peu le tourbillon du monde. C'est dire que la multiplicité des expériences, des sensations et des impressions converge aussi vers un travail d'assomption où se nouent ensemble un désir et un regard, creusant ainsi le sillon secret de l'individualité. C'est d'ailleurs en cela que la Recherche s'élabore formellement depuis un imaginaire et une poétique de la consistance:

D'ailleurs, comme les individualités (humaines ou non) sont dans un liure faites d'impressions nombreuses qui, prises de bien des jeunes filles, de bien des églises, de bien des sonates, servent à faire une seule sonate, une seule église, une seule jeune fille, ne ferais-je pas mon livre de la façon que Françoise faisait ce bøuf mode, apprécié par M. de Norpois, et dont tant de morceaux de viande ajoutés et choisis enrichissaient la gelée. (TR, 340)

Pareils à la gelée du bouf mode, les objets épars de l'expérience trouvent enfin cette consistance qui les fait tenir ensemble, c'est-à-dire les donne à voir selon les lignes reconfigurantes d'un désir. De la même manière, on peut dire que la poétique du texte proustien retrouve ici, dans son ensemble, la problématique de l'origine et du sens. Si l'origine est en effet cet abîme que le NOM vient voiler pour le symboliser soit comme plénitude à venir (Dieu), soit comme abîme de non-sens ou de mystère, on peut dire que l'expérience proustienne de l'écriture s'est aussi mesurée à cet abîme originaire, édifiant, par-delà le vertige devant le sans-fond, la bordure minimalement consistante d'un nom, d'une parole, ou d'un regard. La gelée de bœuf mode - le nom n'est-elle pas la consistance première qui s'élève magiquement au-dessus de l'abîme? De cet abîme, nous ne savons rien, puisque l'origine nous est justement voilée par le nom, la gelée originaire qui nous permet de persister au-dessus de cet inconnu radical, ou de cet «effroyablement ancien", pour le dire comme Blanchot ${ }^{18}$.

Le nom de l'origine n'étant qu'un voile déposé sur l'inconnu, celui-ci trace en définitive la limite - ce que les anciens appelaient déjà Péras dans son opposition à Apeiron - depuis laquelle sens et non-sens sont posés; et c'est parce que l'origine est radicalement inaccessible qu'il y a le commencement, le tracé de fondation, le sillon, ou le nom depuis lequel un sujet se mesure à l'inconnu ou à l'impossible ${ }^{19}$. 
réfléchie - que s'il en est différencié - forme passive. En étant appelé par son nom - qui est et qui n'est pas son corps - il est délivré de son identification imaginaire au réel: il devient sujet. [...] Appeler un enfant du nom qui le désigne, c'est introduire dans le réseau des signes une rupture: un nom propre ne désigne rien parmi les choses représentées" (1974: 128). Le nom propre, comme le trait unaire (tel que l'élabore Lacan), est donc cette trace de pure différence sans laquelle le sujet ne saurait se différencier (risquant de se perdre dans le miroir infini de ses identifications). Cet ancrage dans le nom est aussi ancrage dans la loi dans la mesure où le nom du père s'inscrit alors en tiers par rapport à la mère, séparant symboliquement l'enfant de celleci, le différenciant pour lui donner statut de sujet.

4. J. Lacan, $1966: 348$.

5. C'est en effet par le jeu de la voix qu'est symbolisée la présenceabsence de la mère ainsi que l'analyse D. Vasse: «En effet, toute séparation plus ou moins prolongée d'avec la mère [...] provoque, par suite de l'assimilation de la substance nourricière et de l'expulsion des excréments, un vide, qui met à nouveau en tension les organes des sens ordonnés à la sphère du subtil. Pleurs, cris, quête du regard et des mains, repérage des bruits... tentent de faire advenir l'objet primordial là même où il a disparu dans le corps. Maîtrisant ainsi l'angoisse de la mort, l'enfant tente de restaurer le sentiment de plénitude corporelle qui connote nécessairement celui de la présence. [...] Réveillé par la faim, le nourrisson imite en jouant avec l'air de son cavum le rythme parolier du discours de la mère, il attache son regard aux couleurs et aux formes qui l'évoquent. Ainsi les indices subtils d'une présence que l'enfant imagine, rendent déjà là la mère encore absente. Ces objets «mamaïsés» (F. Dolto) investis par l'enfant diffèrent pour un temps son angoisse et l'apaisent. Ainsi, ce qui est éprouvé comme manque substantiel du corps est identiquement dans le cœur de l'enfant annonce et demande de la présence de l'autre. Au corps à corps se substitue le corps à cœur. Le repérage d'une couleur, la répétition d'un son, l'imitation d'un mouvement, l'hallucination d'une odeur, rétablissent imaginairement l'objet primordial manquant» (1974: 87-

88). C'est en ce sens que la rêverie proustienne sur les noms évoque ce temps de supposée plénitude alors que la voix (par le babil des signifiants) veut se confondre au corps de la mère.

6. Mon analyse ne s'attarde pas ici à déchiffrer l'incidence subtile ou secrète du signifiant sur le signifié. Citons, parmi les nombreuses études qui traitent de cette question, celles de R. Barthes (1972); N. Deschamps (1996); S. Gaubert (1980); G. Genette (1976); J. Milly (1983); A. Henry (1981). Ma lecture cherche plutôt à expliciter le processus de désillusion du Narrateur en montrant, non seulement l'importance symbolique de l'épisode avec Gilberte, mais celle aussi de la poétique du texte proustien qui, entre impressionnisme et géométrisation du regard, ouvre la voie à une autre conception du nom (du langage) et, par conséquent, de la conscience.

7. S. Freud, 1981.

8. S. Freud, 1965

9. Dans les sociétés anciennes, nommer l'autre, c'est aussi avoir pouvoir sur lui; c'est pourquoi le nom véritable est très souvent secret. Posséder le nom, c'est posséder l'être. Cf. J. Markale, 1983: 29-39.

10. Cette écriture-adoration du nom de l'Aimée n'est pourtant pas celle que découvre le Narrateur de la Recherche ainsi qu'on peut justement en lire l'événement dans ce segment du Temps retrouvé (Folio/Classique, 1990) que l'on désigne généralement sous le titre «L'Adoration perpétuelle» (161-224). Récit dans lequel, notamment, il y raconte sa découverte d'un art qui s'inscrit dans une conception particulière de la mémoire et du corps (du «temps incorporé»): «On peut faire se succéder indéfiniment dans une description les objets qui figuraient dans le lieu décrit, la vérité ne commencera qu'au moment où l'écrivain prendra deux objets différents, posera leur rapport, [...] et les enfermera dans les anneaux nécessaires d'un beau style. Même, ainsi que la vie, quand en rapprochant une qualité commune à deux sensations, il dégagera leur essence commune en les réunissant l'une et l'autre pour les soustraire aux contingences du temps, dans une métaphore» (196). En ce sens, on peut dire, à la suite de G. Genette dans "L'âge des noms " (1976), que le romanesque proustien s'élabore aussi à se défaire de cette croyance originaire où nommer, c'est créer et posséder les êtres et les choses. Par ailleurs, il est intéressant de noter que dans cette théorie de l'art, quelque chose de la logique du signifiant se laisse dire, alors que, justement, un signifié ne fait sens que sur la toile de fond du signifiant (donc de la mémoire, du passé, de l'inconscient, de la jouissance).

11. M. Proust, Le Temps retrouvé, p. 179.

12. J.-F. Mattéi, 1996: 287.

13. On retrouve en effet le jeu de cette alternance (consistance et dissémination) dans toute la trame imaginaire de la Recherche ainsi qu'a pu l'analyser J.-P. Richard, 1974. De même, L. Fraisse (1988) a montré l'importance du vitrail - donc de la ligne, de la découpe - dans l'élaboration de cette vision poétique qui n'est pas faite que d'impressions. Enfin, citons l'étude de C. Bouazis (1992), où, se situant à la frontière de la sémiotique et de la psychanalyse, l'auteur montre l'importance première et fondamentale du «trait " contre toute confusion originaire. On pourrait dire à notre tour que le texte proustien est semblable à une bande de Möbius dans la mesure où cette écriture s'inscrit dans un double procès - du nébuleux à la ligne tout en ayant qu'une seule face et un seul bord, là où l'endroit rejoint l'envers, comme l'inconscient rejoint le conscient; texte möbianisé en effet puisque s'y enchevêtrent, par tout un réseau de figures et d'images, les deux temps du sujet (qui oscille entre le nébuleux et le cristal, le non-savoir et l'assomption). Peut-être faut-il lire d'ailleurs dans la célèbre description des carafes de la Vivonne - «[...] dans cette allitération perpétuelle entre l'eau sans consistance où les mains ne pouvaient la capter et le verre sans fluidité où le palais ne pouvait en jouir" (Du côté de chez Swann, p.166) -, la figure de ce texte qui combine non seulement fluidité et ductilité, fumée et cristal, indiscernabilité et discernabilité, mais rêve aussi en cette "allitération perpétuelle", quelque chose comme le premier et perpétuel chant du monde, celui de ce babil originaire qui voudrait se confondre avec le corps de la mère.

14. M. Proust, Le Temps retrouvé, p. 338.

15. J. Derrida, 1972: 247-324.

16. Du mur-miroir à la ligne du train, du balcon baigné de soleil à la bille d'agate, en passant par le nom de Gilberte et le petit bleu, le texte se déploie ainsi selon cette alternance de lignes discernables et indiscernables, miroir de la quête identitaire du Narrateur. La suite du texte n'échappe pas à cette logique alors qu'après avoir évoqué le plan des rues de Paris (en écho au guide horaire du train et au paradigme du repérage), le chapitre se termine sur la déambulation de Madame Swann, mère de Gilberte, dans l'allée des Acacias du Bois de Boulogne. La description du parc y est aussi lisible selon les deux codes, impressionniste et géométrisant: feuilles mortes, tourbillons de taches jaunes, rayon de soleil découpant les feuillages, allées traçant quelques lignes dans le paysage. Mais sans doute et surtout faut-il lire, dans l'élégante parade de Madame Swann, ce qui justement tranche dans le décor, traçant la ligne séduisante d'une épiphanique beauté. L'élégance est ici encore un art de la ligne, du trait, du pli; art de la coupe, de la 
découpe, dont la robe, on l'a dit, est l'un des paradigmes du roman (de l'écriture). À cet égard, on peut dire que le texte de Proust est tout autant proche de l'impressionnisme que du cubisme.

17. Rappelons-nous à cet égard cette "première" page d'écriture du Narrateur citée au début de la Recherche où celui-ci cherche à déchiffrer l'énigme fascinante qu'exercent sur lui, alors qu'il se promène en voiture dans la campagne, les diverses visions qu'il a des Clochers de Martinville. Expérience vertigineuse qui se termine ainsi : "Je ne repensai jamais à cette page, mais à ce moment-là, quand, [...] j'eus fini de l'écrire, je me trouvai si heureux, je sentais qu'elle m'avait si parfaitement débarrassé de ces clochers et de ce qu'ils cachaient derrière eux, que comme si j'avais été moi-même une poule et si je venais de pondre un œuf, je me mis à chanter à tue-tête» (180). Ce récit explicite évidemment le perspectivisme de toute perception ou de tout regard, soulignant avec force que le sujet est toujours en mouvement, reconfigurant ainsi un objet qui semble échapper à toute définition stable. Mais, il en énonce aussi le chiffre ou la règle, ce qui est sans doute une manière de se déprendre un tant soit peu de cette scène, en décrivant le mécanisme de cette fascination. Écrire, en ce sens, c'est littéralement "se débarrasser de ces clochers", c'est-à-dire déchiffrer l'énigme de ce regard qui pourrait se perdre dans cette fascination. C'est là encore ce geste ancien et premier qui s'assure de cette maîtrise minimale sans laquelle cependant le tourbillon du monde ne pourrait pas même être appréhendé comme tel. Cette joie, enfin, est celle qui vient au sujet en même temps qu'il sait un tant soit peu nommer le monde. Cette page d'écriture - première leçon de chose - est comme encryptée dans le texte de la Recherche; trace ancienne de ce qui n'était pas encore un roman, mais une sorte d'épiphanie libératrice. Le roman ayant ainsi sa scène d'écriture originaire, pur surgissement, avant que ne s'élabore la patiente quête du temps retrouvé

18. J'ai déjà eu l'occasion d'analyser cette problématique de l'origine chez Blanchot: J. Cardinal, «Du (post)modernisme comme deuil.

L'éthique de l'anonymat chez Maurice Blanchot", Études littéraires, vol. 27, no 1, été 1994; et «Agonie et passion dans Thomas l'Obscur de Maurice Blanchot", Biffures, no 1, 1997. J'ai également abordé la question de l'origine dans mon article "De l'Un à l'Autre. Le désir de savoir et l'écriture de la différence dans La recherche de l'absolu de Balzac",Texte, no 17, 1995.

19. D'une origine à l'autre - de la conscience à celle du repérage du sujet dans le cosmos - ne retrouve-t-on pas en effet le même processus identitaire dans la mesure où l'on passe du magma à la ligne, de Apeiron (illimité, indéfini) à Péras (limite), premier schéma ou logique première qui fonde le sujet. À cet égard, voir la démonstration de J.-F. Mattéi (1996). De même, M. Serres retrouve cette intuition première dans son livre sur Les Origines de la géométrie (1993). Contre une doxa poststructuraliste qui affirme dans le même souffle le sans-fond de l'origine et l'impossible constitution d'un sujet (ouvert désormais sur toutes les identités), il est peut-être bon de rappeler que si la métaphysique du propre est bien en partie un leurre, il n'en reste pas moins vrai que c'est justement parce que cet absolu (de la fondation ou du nom propre) n'existe pas que le sujet est appelé à se nommer (en nommant le monde), pour mieux surmonter le vertige de la béance originaire.

\section{RÉfÉRENCES BIBLIO G RAPHIQ U ES}

BARTHES, R. [1972]: "Proust et les noms", Le Degré zéro de l'écriture, Paris, Seuil, coll. «Points».

BouAzIs, C. [1992]: Ce que Proust savait du symptôme, Paris, Méridiens/ Klincksieck.

Compagnon, A. [1989]: Proust entre deux siècles, Paris, Seuil.

Deleuze, G. [1970]: Proust et les signes, Paris, P.U.F.

DerRIDA, J. [1972]: «La mythologie blanche», Marges (de la philosophie), Paris, Minuit, coll. "Critique».

Deschamps, N. [1996]: «L'Innommable des noms proustiens», Le Texte et le Nom (collectif), Montréal, XYZ éditeur.

Fraisse, L. [1988] : Le Processus de la création chez Proust, Paris, José

Corti.

FREUD, S. [1981]: «Au-delà du principe de plaisir», Essais de

psychanalyse, Paris, Petite Bibliothèque Payot;

[1965]: «Animisme, magie et toute-puissance des idées», Totem et

Tabou, Paris, Petite Bibliothèque Payot.

GauberT, S. [1980]: «Le jeu de l'Alphabet», Recherche de Proust

(collectif), Paris, Seuil, coll. «Points».

GenetTe, G. [1972]: Figures III, Paris, Seuil;

[1976]: «L'âge des noms», Mimologiques, Paris, Seuil, coll. « Poétique».

HenRY, A. [1981]: «Les noms, les mots, les choses", Marcel Proust.

Théories pour une esthétique, Paris, Klincksieck.

KRISTEVA, J. [1994] : Le Temps sensible, Paris, Gallimard.

LACAN, J. [1966]: Écrits, Paris, Seuil, coll. «Le champ freudien».

LatTre, A. de [1978-85]: La Doctrine de la réalité chez Proust (3 vol.),

Paris, José Corti.

MARKALE, J. [1983]: «Le nom, la parole et la magie», Corps écrit (Le

Nom), no 8, Paris, P.U.F.

MATTÉI, J.-F. [1996]: Platon et le miroir du mythe, Paris, P.U.F., coll.

"Thémis/Philosophie».

Milly, J. [1983]: La Phrase de Proust, Paris, Champion, coll.

«Unichamp».

RichARD, J.P. [1974]: Proust et le monde sensible, Paris, Seuil, coll.

«Points".

SERres, M. [1993]: Les Origines de la géométrie, Paris, Flammarion, coll.

"Champs".

TADiÉ, J.-Y. [1986]: Proust et le roman, Paris, Gallimard, coll. «Tel» VAsse, D. [1974]: L'Ombilic et la Voix, Paris, Seuil, coll. «Le champ freudien". 